\title{
A First Course
} in Module Theory 
This page is intentionally left blank 


\section{A First Course}

\section{in Module Theory}

\section{E Keating \\ Imperial College, London}




\title{
Published by
}

Imperial College Press

203 Electrical Engineering Building

Imperial College

London SW7 2BT

\section{Distributed by}

World Scientific Publishing Co. Pte. Ltd.

P O Box 128, Farrer Road, Singapore 912805

USA office: Suite 1B, 1060 Main Street, River Edge, NJ 07661

UK office: 57 Shelton Street, Covent Garden, London WC2H 9HE

\author{
Library of Congress Cataloging-in-Publication Data \\ Keating, M. E., 1941- \\ A first course in module theory / M. E. Keating. \\ p. $\mathrm{cm}$. \\ Includes bibliographical references and index \\ ISBN 186094096X (alk. paper) \\ 1. Modules (Algebra) \\ QA247.K43 1998 \\ 512'.4--dc21

\section{British Library Cataloguing-in-Publication Data} \\ A catalogue record for this book is available from the British Library.
}

\section{Copyright (C) 1998 by Imperial College Press}

All rights reserved. This book, or parts thereof, may not be reproduced in any form or by any means, electronic or mechanical, including photocopying, recording or any information storage and retrieval system now known or to be invented, without written permission from the Publisher.

For photocopying of material in this volume, please pay a copying fee through the Copyright Clearance Center, Inc., 222 Rosewood Drive, Danvers, MA 01923, USA. In this case permission to photocopy is not required from the publisher.

This book is printed on acid-free paper.

Printed in Singapore by Uto-Print 


\author{
To \\ Valerie \\ and \\ Christopher
}


This page is intentionally left blank 


\section{Introduction}

The purpose of this book is to provide an introduction to module theory for a reader who knows something of linear algebra and elementary ring theory.

There is a very natural theme for a first course in module theory, namely the structure theory of modules over Euclidean domains. This theory is very explicit, and it has interesting and surprisingly disparate interpretations. An abelian group can be regarded as a module over the ring of integers $\mathbb{Z}$, while a matrix with entries in a field $F$ defines a module over the polynomial ring $F[X]$. As both $\mathbb{Z}$ and $F[X]$ are examples of Euclidean domains, the general theory of modules over Euclidean domains leads to specific results about abelian groups and about matrices. In the former, we obtain a classification of finitely generated abelian groups, and, in the latter, a description of the rational canonical form and the Jordan normal form of a matrix.

Although the structure theory for modules over Euclidean domains is the core of this text, we also consider modules over more general, even noncommutative, rings of coefficients. This extra generality allows us to discuss the limitations and some of the extensions of our main results.

The contents of this text are based on a final year undergraduate course that I gave a number of times at Imperial College, London, with some additional material. In the lecture course, I assumed that everyone was familiar with the elementary properties of rings, ideals and Euclidean domains. Here I have provided an introduction to ring theory in the first two chapters, so that the text is more self-contained than the lectures, and a greater variety of rings can be used.

Chapters 3 to 7 expound the basics of module theory, including methods of comparing, constructing and decomposing modules. The results in these chapters are rather general and do not depend much on the ring of coefficients. Chapters 8 to 12 are the heart of this text, since it is here that we obtain the strong results that are special to Euclidean domains. Chapter 12 also contains two applications of the theory, to abelian groups 
and to lattices.

In Chapter 13, we use the module theory to find two standard forms for a square matrix, namely, the rational canonical form and the Jordan normal form of a matrix. In addition to the usual version of the Jordan normal form of a matrix over the complex numbers, we give two further versions that apply to a matrix whose entries are taken from a field other than the complex numbers. The second of these variations is used, without proof, in a fundamental paper on representation theory by $\mathbf{J}$ A Green [Green], and a proof has not been published before at an elementary level, as far as I know. I am grateful to my colleague Gordon James for drawing my attention to these versions of the Jordan normal form.

In the final chapter, we go beyond the bounds of Euclidean domains to look at some basic results on projective modules over rings in general. Here, we establish some basic facts about group algebras and the relationship between module theory and the representation theory of groups.

As befits a book that is intended as a first course for undergraduates, arguments are given in considerable detail, at least, in the earlier part of text. There is an index entry "proofs in full detail" to help the reader to locate these agruments. There are many explicit illustrations and exercises, and some hints and partial solutions to the exercises are provided in an appendix.

Material that was not covered in the original lecture course is indicated $\mathbf{S}$ by a "supplementary" symbol as in the margin. This material in not essential for the core results on modules over Euclidean domains, and it can be omitted if the reader wishes.

Each chapter has a section headed "Further developments" in which the definitions and results of the chapter are placed in a wider setting. References are provided to enable the interested reader to follow up the topics that are introduced in these sections. I hope that these sections will provide a useful source of projects for students who are in the final year of a four-year undergraduate MMath or MSci course at a UK university, or who are taking the new one-year preliminary research degree, the "MRes".

The text is divided into chapters and sections, which are numbered as you would expect: 1.1, 1.2, and so on. For ease of reference, results are numbered consecutively within each section, and so they appear in the form "12.1.1 Theorem", "12.1.2 Lemma", etc.

This book was written while I was also working on the more advanced texts [B \& K: IRM] and [B \& K: CM] with Jon Berrick of the National University of Singapore. My collaboration with Jon has had many influences, both on my teaching of the lecture course and on the composition of this text, which it is impossible to acknowledge individually. 
Finally, I should thank the Mathematics Department at Imperial for allowing me the time and space in which to write textbooks. A special thanks also to my colleagues at Imperial for their assistance in mastering $\operatorname{LAT}_{\mathrm{E}} \mathrm{X}$. Phillip Kent of the METRIC Project ran an introductory course which got me started, and Oliver Pretzel provided me with the $\mathrm{LAT}_{\mathrm{E}} \mathrm{X}$ software, and kindly and patiently explained why it sometimes did not do what I hoped. 
This page is intentionally left blank 


\section{Contents}

Introduction $\quad$ vii

1 Rings and Ideals $1 \ldots \ldots$

1.1 Groups . . . . . . . . . . . . . . . . . 1

$1.2 \quad$ Rings . . . . . . . . . . . . . . . . . . 3

1.3 Commutative domains . . . . . . . . . . . . . 4

1.4 Units . . . . . . . . . . . . . . . . 4

$1.5 \quad$ Fields . . . . . . . . . . . . . . . . . 5

1.6 Polynomial rings . . . . . . . . . . . . . . . . . . 5

1.7 Ideals . . . . . . . . . . . . . . . . . . 7

1.8 Principal ideals $\ldots \ldots \ldots \ldots \ldots$

1.9 Sum and intersection . . . . . . . . . . . . . 9

1.10 Residue rings . . . . . . . . . . . . . . . 10

1.11 Residues of integers . . . . . . . . . . . . . . 12

Exercises . . . . . . . . . . . . . . . . . . . 14

2 Euclidean Domains 17

2.1 The definition . . . . . . . . . . . . . 17

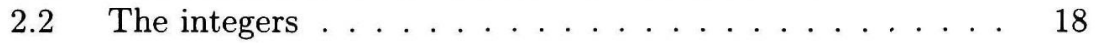

2.3 Polynomial rings. . . . . . . . . . . . . . . . . . . 18

2.4 The Gaussian integers $\ldots \ldots \ldots \ldots$

2.5 Units and ideals . . . . . . . . . . . . . 20

2.6 Greatest common divisors . . . . . . . . . . . . . . 21

2.7 Euclid's algorithm . . . . . . . . . . . . . . . . 22

2.8 Factorization . . . . . . . . . . . . . . . . . . 23

2.9 Standard factorizations . . . . . . . . . . . . . 25

2.10 Irreducible elements . . . . . . . . . . . . . . . 26

2.11 Residue rings of Euclidean domains . . . . . . . . . 28

2.12 Residue rings of polynomial rings $\ldots \ldots \ldots \ldots \ldots$

2.13 Splitting fields for polynomials . . . . . . . . . . . 31 
2.14 Further developments . . . . . . . . . . . . . . 31

Exercises ...................... 32

3 Modules and Submodules $\quad 35$

3.1 The definition . . . . . . . . . . . . . . 35

3.2 Additive groups . . . . . . . . . . . . . . . . . . . 37

3.3 Matrix actions . . . . . . . . . . . . . . . . 37

3.4 Actions of scalar matrices . . . . . . . . . . . . . . . 39

3.5 Submodules . . . . . . . . . . . . . . . . . . 40

3.6 Sum and intersection ................ 41

$3.7 \quad k$-fold sums . . . . . . . . . . . . . . . . . . . 43

3.8 Generators . . . . . . . . . . . . . . . . . . 43

3.9 Matrix actions again . . . . . . . . . . . . 45

3.10 Eigenspaces . . . . . . . . . . . . . . . . . 46

3.11 Example: a triangular matrix action . . . . . . . . . 47

3.12 Example: a rotation . . . . . . . . . . . . . . . . . 48

Exercises . . . . . . . . . . . . . . . . . . . 48

4 Homomorphisms $\quad 51$

4.1 The definition ...................... 51

4.2 Sums and products ................... 53

4.3 Multiplication homomorphisms . . . . . . . . . . 54

$4.4 \quad F[X]$-modules in general . . . . . . . . . . . 55

$4.5 \quad F[X]$-module homomorphisms . . . . . . . . 56

4.6 The matrix interpretation . . . . . . . . . . 57

4.7 Example: $p=1 \ldots \ldots \ldots$. . . . . . . . . . 57

4.8 Example: a triangular action . . . . . . . . . . . . 58

4.9 Kernel and image . . . . . . . . . . . . . . . . . 58

4.10 Rank \& nullity . . . . . . . . . . . . . . . . . 60

4.11 Some calculations . . . . . . . . . . . . . . . 61

4.12 Isomorphisms . . . . . . . . . . . . . . . . . . 62

4.13 A submodule correspondence . . . . . . . . . . . 64

Exercises . . . . . . . . . . . . . . . . . 66 65

5 Free Modules $\quad 69$

5.1 The standard free modules . . . . . . . . . . . . . 70

5.2 Free modules in general . . . . . . . . . . . . . . . 71

5.3 A running example .................. . . 73

5.4 Bases and isomorphisms .................. 74

5.5 Uniqueness of rank . . . . . . . . . . . . 76

5.6 Change of basis . . . . . . . . . . . . . 77

5.7 Coordinates . . . . . . . . . . . . . . 79 
5.8 Constructing bases ................. . . 80

5.9 Matrices and homomorphisms . . . . . . . . . . 81

5.10 Illustration: the standard case . . . . . . . . . . . 83

5.11 Matrices and change of basis . . . . . . . . . . . . 84

5.12 Determinants and invertible matrices . . . . . . . . 85

Exercises . . . . . . . . . . . . . . . . 88

6 Quotient Modules and Cyclic Modules $\quad 91$

6.1 Quotient modules ................. 92

6.2 The canonical homomorphism . . . . . . . . . . . 92

6.3 Induced homomorphisms . . . . . . . . . . . . . . . . 93

6.4 Cyclic modules . . . . . . . . . . . . . . . . . . 94

6.5 Submodules of cyclic modules . . . . . . . . . . . . 96

6.6 The companion matrix . . . . . . . . . . . . . . . . . . 99

6.7 Cyclic modules over polynomial rings . . . . . . . . . . 100

6.8 Further developments . . . . . . . . . . . . . . . . . . . . . 102

Exercises . . . . . . . . . . . . . . . . . . . 102

7 Direct Sums of Modules $\quad 107$

7.1 Internal direct sums . . . . . . . . . . . . . . . . 107

7.2 A diagrammatic interpretation . . . . . . . . . . . 109

7.3 Indecomposable modules . . . . . . . . . . . . . . . . . . 111

7.4 Many components . . . . . . . . . . . . . . . . . 112

7.5 Block diagonal actions . . . . . . . . . . . . . 113

$7.6 \quad$ External direct sums . . . . . . . . . . . . . . . . . 114

7.7 Switching between internal \& external . . . . . . . . . . . 115

7.8 The Chinese Remainder Theorem . . . . . . . . . . . . . . 116

Exercises . . . . . . . . . . . . . . . . . . . 118

8 Torsion and the Primary Decomposition 123

8.1 Torsion elements and modules . . . . . . . . . . . . . . 124

8.2 Annihilators of modules . . . . . . . . . . . . . 125

8.3 Primary modules . . . . . . . . . . . . . . . 126

8.4 The $p$-primary component . . . . . . . . . . . . . 128

8.5 Cyclic modules . . . . . . . . . . . . . . 130

8.6 Further developments . . . . . . . . . . . . . . . 130

Exercises . . . . . . . . . . . . . . . . . 131

9 Presentations $\quad 133$

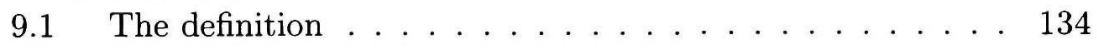

9.2 Relations .................... . 135

9.3 Defining a module by relations . . . . . . . . . . 136 
9.4 The fundamental problem . . . . . . . . . . . . . . 136

9.5 The presentation matrix . . . . . . . . . . . . 139

9.6 The presentation homomorphism . . . . . . . . . 140

$9.7 F[X]$-module presentations . . . . . . . . . . . . 141

9.8 Further developments . . . . . . . . . . . . . . . . . 142

Exercises . . . . . . . . . . . . . . . . . . 143

10 Diagonalizing and Inverting Matrices 145

10.1 Elementary operations . . . . . . . . . . . . . . 145

10.2 The effect on defining relations . . . . . . . . . 146

10.3 A matrix interpretation . . . . . . . . . . . . . . . 149

10.4 Row \& column operations in general . . . . . . . . . . . 150

10.5 The invariant factor form . . . . . . . . . . . . . . 152

10.6 Equivalence of matrices . . . . . . . . . . . . . . 155

10.7 A computational technique . . . . . . . . . . . 156

10.8 Invertible matrices . . . . . . . . . . . . . . . 158

10.9 Further developments . . . . . . . . . . . . . . 160

Exercises . . . . . . . . . . . . . . . . 161

11 Fitting Ideals $\quad 163$

11.1 The definition . . . . . . . . . . . . . 163

11.2 Elementary properties . . . . . . . . . . . . . 165

11.3 Uniqueness of invariant factors . . . . . . . . . . . 166

11.4 The characteristic polynomial . . . . . . . . . . 167

11.5 Further developments . . . . . . . . . . . . . . . . 168

Exercises . . . . . . . . . . . . . . . . 169

12 The Decomposition of Modules $\quad 171$

12.1 Submodules of free modules . . . . . . . . . . . . . 171

12.2 Invariant factor presentations . . . . . . . . . . . 174

12.3 The invariant factor decomposition . . . . . . . . . 176

12.4 Some illustrations . . . . . . . . . . . . . . . . 178

12.5 The primary decomposition . . . . . . . . . . 179

12.6 The illustrations, again . . . . . . . . . . . . 181

12.7 Reconstructing the invariant factors . . . . . . . . . . 182

12.8 The uniqueness results . . . . . . . . . . . . . 183

12.9 A summary . . . . . . . . . . . . . . . . . . 185

12.10 Abelian groups . . . . . . . . . . . . . . 186

12.11 Lattices . . . . . . . . . . . . . . . . . . 187

12.12 Further developments . . . . . . . . . . . . . . 190

Exercises . . . . . . . . . . . . . . . . . . 190 
13 Normal Forms for Matrices $\quad 193$

$13.1 F[X]$-modules and similarity . . . . . . . . . . . . 194

13.2 The minimum polynomial . . . . . . . . . . . 195

13.3 The rational canonical form . . . . . . . . . . . . . 197

13.4 The Jordan normal form: split case . . . . . . . . . . . 200

13.5 A comparison of computations . . . . . . . . . . . . . 202

13.6 The Jordan normal form: nonsplit case . . . . . . . . . . . 203

13.7 The Jordan normal form: separable case . . . . . . . . . . 206

13.8 Nilpotent matrices . . . . . . . . . . . . . . . . . . . . . . 209

13.9 Roots of unity . . . . . . . . . . . . . . . . . 209

13.10 Further developments . . . . . . . . . . . . . . 211

Exercises . . . . . . . . . . . . . . . . 211

14 Projective Modules $\quad 215$

14.1 The definition . . . . . . . . . . . . . . 215

14.2 Split homomorphisms . . . . . . . . . . . . 216

14.3 Semisimple rings . . . . . . . . . . . . . . . 220

14.4 Representations of groups . . . . . . . . . . . . . 222

14.5 Hereditary rings . . . . . . . . . . . . . . . 224

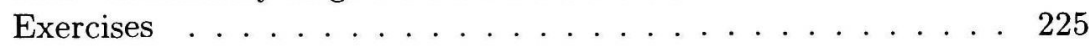

$\begin{array}{lr}\text { Hints and Solutions } & 229\end{array}$

Bibliography $\quad 243$

$\begin{array}{ll}\text { Index } & 245\end{array}$ 